\title{
ELECTRON PROPERTIES IN GaAS FOR THE DESIGN OF MM-WAVE IMPATTS
}

\author{
Heribert Eisele \\ Center for High-Frequency Microelectronics \\ Department of Electrical Engineering \& Computer Science \\ The University of Michigan \\ 2245 EECS Building \\ Ann Arbor, Michigan 48109-2122
}

Received February 16, 1991

\begin{abstract}
A very staightforward method has been developed to apply space-charge resistance measurements for determining the high-field drift velocity of electrons in GaAs. The breakdown voltages of the single-drift flat-profile IMPATT diodes used in these measurements justify the validity of well known ionization rates for still higher electric fields.
\end{abstract}

\section{Introduction}

GaAs IMPATT diodes for frequencies around $94 \mathrm{GHz}$ and higher ${ }^{1}$ operate in the active region with a maximum electric field $E$ above $800 \mathrm{kVcm}^{-1}$. At these fields any systematic experimental values for drift velocities $v_{d n}, v_{d p}$ and ionization rates $\alpha_{n}, \alpha_{p}$ of electron and holes especially at elevated temperatures have not been reported. So far, two methods have been used to determine the drift velocity of electrons in $\mathrm{GaAs}$, the time-of-flight method ${ }^{2-5}$ giving very precise data only up to $234 \mathrm{kVcm}^{-1}$ and the method using the space-charge resistance ${ }^{6} \boldsymbol{R}_{s c}$. Neither the well known equation for the space-charge resistance ${ }^{7}$ nor a more sophisticated method ${ }^{8}$ take into account that $v_{d n}$ monotonically decreases for high electric fields according to theoretical ${ }^{9-11}$ and experimental results ${ }^{2-6}$.

Since a short and well defined avalanche region does not exist for single-drift W-band IMPATT diodes as already discussed ${ }^{12}$, the avalanche region considerably influences $R_{s c}$. Therefore a very straightforward method has been developed to calculate $R_{s c}$ from the basic continuity and Poisson's equations using different velocity vs. field profiles. 


\section{Static approximation for the space-charge resistance}

In order to include the effect of the avalanche region it is assumed that $R_{s c}$ measured at $f$ above the thermal cut-off frequency can be derived from the static I/V-characteristic excluding thermal effects,

$$
R_{s c}=\left.\frac{d V}{d I}\right|_{I=I_{B}}
$$

where $I_{B}$ is the bias current.

If diffusion effects are neglected in the space-charge region, the equation for the electron current density $J_{n}$ as a function of space $x$ from $-w_{p}$ to $w_{n}$, bias current $I_{B}$ and active device area $A_{D}$ can be written as ${ }^{6}$

$$
\frac{J_{n}(x) A_{D}}{I_{B}}=\exp \int_{-w_{p}}^{w_{n}}\left[\alpha_{n}(x)-\alpha_{p}(x)\right] d x \cdot \int_{-w_{p}}^{w_{n}} \alpha_{p}(x) \exp \int_{-w_{p}}^{x}\left[\alpha_{p}\left(x^{\prime}\right)-\alpha_{n}\left(x^{\prime}\right)\right] d x^{\prime} d x
$$

with $\alpha_{n}(x)=\alpha_{n}(E(x))$ and $\alpha_{p}(x)=\alpha_{p}(E(x))$.

The most recent paper on ionization rates ${ }^{13}$ includes a dead space correction ${ }^{14}$ for an energy below the threshold $W_{t h}$ in the dead space region of length $d$ ( $q$ : electron charge).

$$
\alpha_{n}(x)=0 \text { for } q \int_{-w_{p}}^{-w_{p}+d} E(x) d x \leq W_{t h}
$$

Eq. (3) was used for the ionization rates where applicable, otherwise $W_{t h}$ was set to zero.

The integration of Poisson's equation gives

$$
E(x)=\frac{q}{\epsilon_{r} \epsilon_{0}} \int_{-w_{p}}^{w_{n}}\left[N_{D}(x)-N_{A}(x)+\frac{J_{c}-J_{n}(x)}{q v_{d p}(x)}-\frac{J_{n}(x)}{q v_{d n}(x)}\right] d x
$$

whereby $v_{d n}(x)=v_{d n}(E(x)), v_{d p}(x)=v_{d p}(E(x)), J_{c} A_{D} \approx I_{B}, N_{D}(x)-N_{A}(x)$ is doping concentration and $\epsilon_{r} \epsilon_{0}$ the semiconductor permittivity. Intergrating eq. (4) and taking the built-in voltage ${ }^{7} V_{b i}$ into account gives the voltage $V$ at the terminals.

$$
V=\int_{-w_{p}}^{w_{n}} E(x) d x-V_{b i}
$$

Eq. (1) to (5) were solved numerically whereby in eq. (4) three different curves for velocity-field dependence were assumed as shown in Fig. 1. At fields below $234 \mathrm{kVcm}^{-1}$ all three were based upon the well matching experimental values ${ }^{2-5}$ in the literature, the values above were generated from a hyperbolic decrease comparable to simulations ${ }^{9,10}$. For the drift velocity of holes $v_{d p}$ experimental values ${ }^{15}$ below $100 \mathrm{kVcm}^{-1}$ and theoretical values ${ }^{16}$ above $100 \mathrm{kVcm}^{-1}$ were applied. Since holes mainly occur in the avalanche region, changes in the velocity 


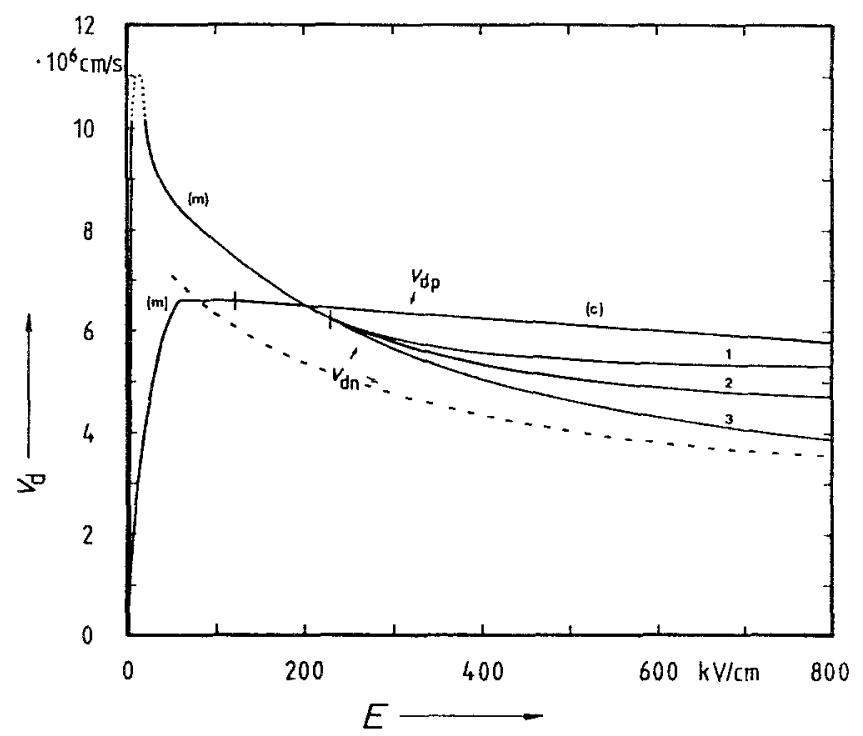

Fig. 1: Drift velocities $v_{d n}, v_{d p}$ of electrons and holes vs. the electric field $E$ -: $T_{0}=300 \mathrm{~K}$

(m): measured characteristics ${ }^{2-5,15}\left(T_{0}=300 \mathrm{~K}\right)$

(c): calculated curve $\left(T_{0}=300 \mathrm{~K}\right)$

- -: extrapolated curve for $T=500 \mathrm{~K}$.

vs. field profile of holes only slightly influence the solution of eq. (1) to (5).

\section{Measurement set-up}

The expected value range for $R_{s c}$ according to eq. (1) lies between about $0.5 \Omega$ and $10 \Omega$. Therefore, a fixture having a low series resistance and series inductance was used to determine the low-frequency (LF) impedances $\underline{Z}_{f}, \underline{Z}_{r}$ of the IMPATT diode in both the forward and reverse (breakdown) directions. The residual series resistance $(<5 \mathrm{~m} \Omega)$ and inductance $(\ll 10 \mathrm{nH})$ were evaluated by replacing the diode with a short and thereafter with a precise resistor and they were factored out for the actual measurement by the computer program.

Fig. 2 shows the schematic circuit diagram of the measurement set-up including the bias circuit for the IMPATT diode $\left(R_{1}, R_{2}, C_{1}, C_{2}\right.$ and $\left.C_{3}\right)$. The reference resistor $R_{3}$ and the IMPATT diode $D$ form a voltage divider, $R_{4}$ and $R_{5}$ ensure decoupling and impedance matching. The low-noise broadband amplifier VV in parallel with the terminals of the diode had an input impedance above $1 \mathrm{k} \Omega$ and was provided to measure the open-circuit noise voltage at the breakdown. 


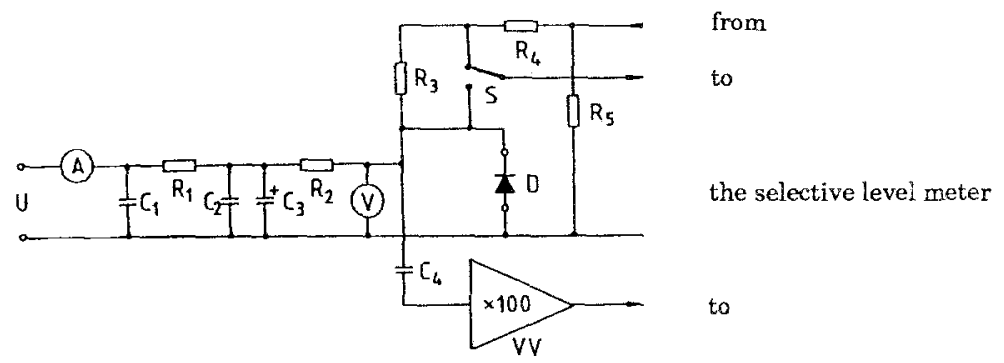

Fig. 2: Schematic diagram of the bias circuit and the impedance evaluation circuit.

D: IMPATT diode

VV: AD 9611

$\begin{array}{llllllll}\mathrm{R}_{1}: 50 \Omega & \mathrm{R}_{2}: 50 \Omega & \mathrm{R}_{3}: 50 \Omega & \mathrm{R}_{4}: 510 \Omega & \mathrm{R}_{5}: 56 \Omega\end{array}$

$\begin{array}{llllll}\mathrm{C}_{1}: & >10 \mu \mathrm{F} & \mathrm{C}_{2}: & 10 \mu \mathrm{F} & \mathrm{C}_{3}:>4700 \mu \mathrm{F} & \mathrm{C}_{4}:>10 \mu \mathrm{F}\end{array}$

\section{Experimental results}

Several ionization rates reported in the literature ${ }^{13}$ were extrapolated to higher electric felds and the calculated breakdown voltages compared to the experimental ones of single-drift flat-profile IMPATT diodes with their abrupt pn-junction. As can be seen in Fig. 3 excellent agreement was found for ionization rates given by Bulman et $\mathrm{al}^{13}$. For this reason these ionization rates and their extrapolations were exclusively used in the following theory-experiment comparision.

Fig. 4 shows the absolute value of the diode impedance $\underline{Z}_{r}$ as a function of frequency $f$. For $f<100 \mathrm{~Hz}\left|\underline{Z}_{r}\right|$ mainly consists of the thermal resistance $R_{t h 1}$ (due to the heat-flow resistance ${ }^{17} r_{w}$ ), the space-charge resistance $R_{s c}$ and the diode series resistance $R_{s}$, but for $f>30 \mathrm{MHz}\left|\underline{Z}_{r}\right|$ reduces to $R_{s c}$ and the diode series resistance $R_{s}$ closely enough. $R_{s}$ was determined in the forward direction for $f>30 \mathrm{MHz}$ where the absolute value of diode impedance $\underline{Z}_{f}$ consists of $R_{s}$, the resistance $R_{u d}$ of the undepleted region $w_{\text {a }}$ and the small-signal impedance $R_{d}$ of the pn-junction. In GaAs the diffusion capacitance can be neglected at these frequencies and the operating bias current $I_{f}$, and, therefore,

$$
\begin{aligned}
R_{d} & =\frac{k T_{j}}{q I_{f}}, \\
R_{u d} & =\frac{w_{s}}{\sigma A_{D}},
\end{aligned}
$$

whereby $\sigma$ is the conductivity in the active region of the diode, $k$ the Boltzmann constant and $T_{j}$ the junction temperature.

It should be noted ${ }^{18}$ that essentially for $f<100 \mathrm{~Hz}$

$$
\underline{Z}_{f}=R_{t h 2}+R_{s}+R_{u d}+R_{d}
$$




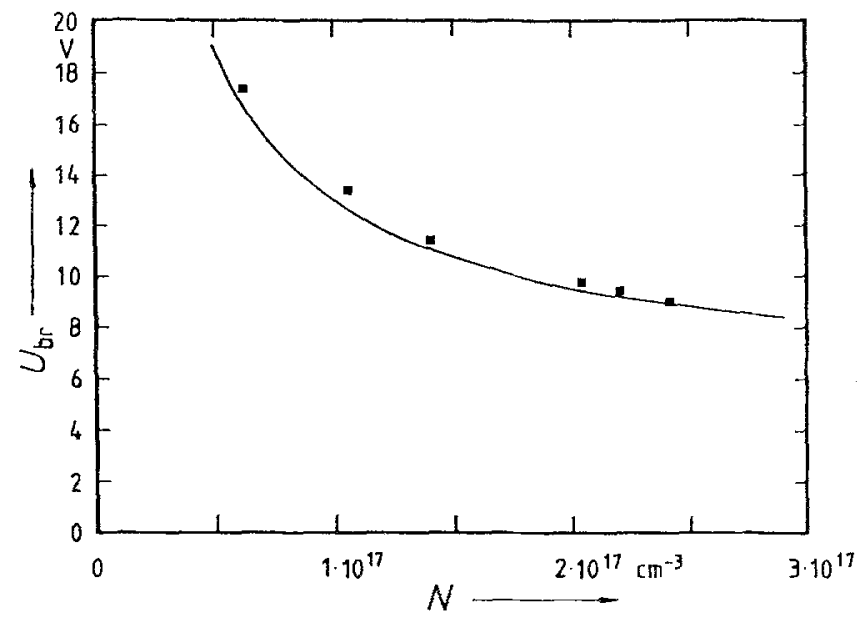

Fig. 3: Breakdown voltage $U_{b r}$ of an abrupt pn-junction as a function of the doping concentration $N$ in the n-type layer.

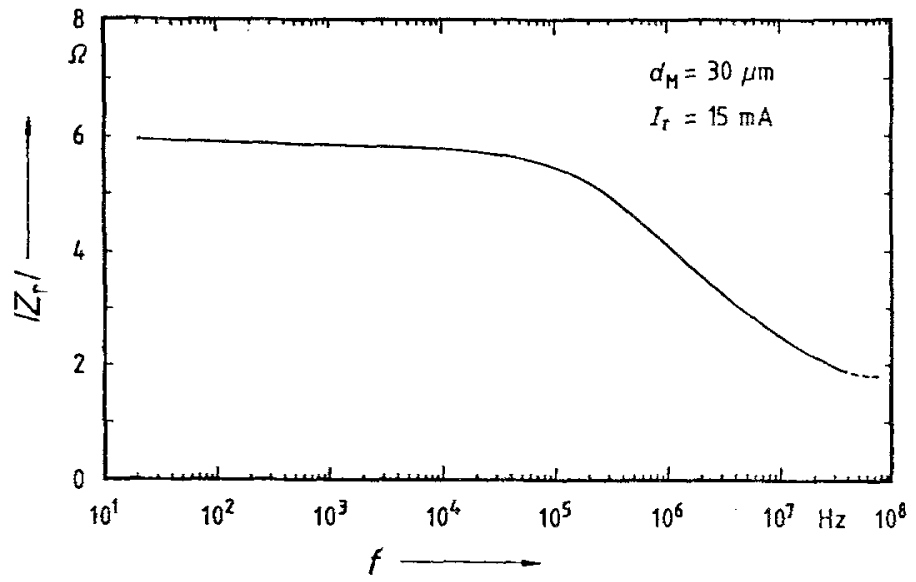

Fig. 4: Absolute value of the small signal diode impedance $\underline{Z}_{r}$ at breakdown $\left(I_{B}=I_{r}\right)$ as a function of the frequency $f\left(d_{M}\right.$ : diode diameter). 
has the additional negative thermal resistance $R_{t h 2}$ due to the heat-flow resistance $r_{w}$ as can be seen in Fig. 5 . $R_{t h 2}$ can be roughly evaluated ${ }^{18}$ (with $\beta_{d}$ : temperature coefficient of the forward voltage $V_{d}$ ) to

$$
R_{t h 2} \approx \beta_{d} r_{w}\left(V_{d}+2 R_{d} I_{f}\right)
$$

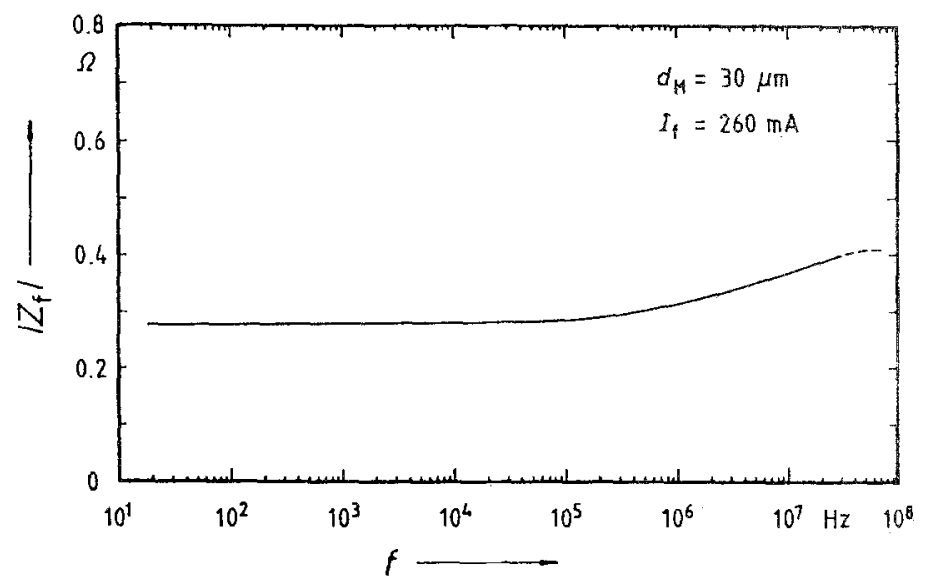

Fig. 5: Absolute value of the small signal diode impedance $\underline{Z}_{f}$ in forward direction at $I_{f}$ as a function of the frequency $f\left(d_{M}\right.$ : diode diameter).

Low-frequency noise measurements ${ }^{17}$ as shown in Fig. 6 were used to characterize the uniformity of the breakdown, and Fig. 7 together with Fig. 6 clearly depicts that uniform breakdown is reached at current densities about $2 \mathrm{kAcm}^{-2}$, which is comparable to values reported before ${ }^{8}$. For this bias the temperature increase is below $10 \mathrm{~K}$ for diodes on diamond heat sinks and below $20 \mathrm{~K}$ for diodes on copper heat sinks and, therefore, it can be neglected. For higher current densities $\left|\underline{Z}_{r}\right|$ increases because the junction temperatures raises, the space-charge region widens ${ }^{12}$, and the current is more displaced off the center to the border of the device area.

Finally Fig. 8 presents $R_{s c}$ as a function of the doping concentration $N_{D}-N_{A}$ in the active region for the three velocity vs. field profiles shown in Fig. 1. The measured space-charge resistances of single-drift flat-profile IMPATT diodes with six different doping levels in the active region ranging from $6.3 \times 10^{16} \mathrm{~cm}^{-3}$ to $2.4 \times 10^{17} \mathrm{~cm}^{-3}$ (Q-band to V-band ${ }^{20,21}, \mathrm{~W}$-band ${ }^{1}$ ) are in excellent agreement with the calculated curve 2 that goes from $6.3 \times 10^{6} \mathrm{cms}^{-1}$ at $215 \mathrm{kVcm}^{-1}$ down to $3.8 \times 10^{6} \mathrm{cms}^{-1}$ at $800 \mathrm{kVcm}^{-1}$ for a temperature of $300 \mathrm{~K}$. Furthermore, this curve also agrees very well with the recent results of a detailed Monte-Carlo simulation ${ }^{11}$. The doping concentration in the active region was determined by 


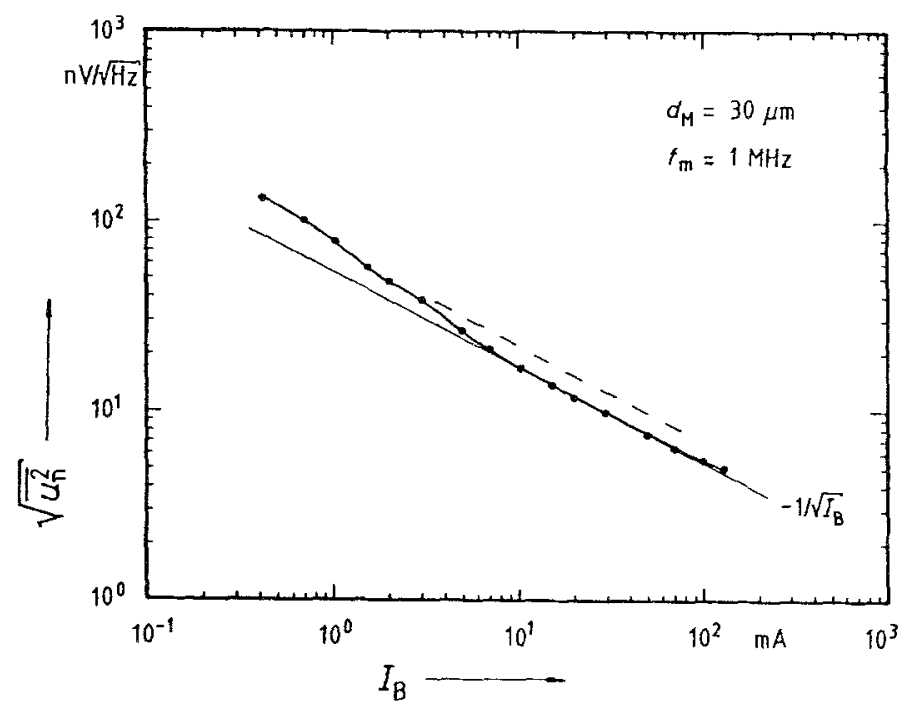

Fig. 6: Open circuit noise voltage per unit bandwidth vs. bias currrent $I_{B}$ at breakdown (measuring frequency $f \approx 1 \mathrm{MHz}$ ).

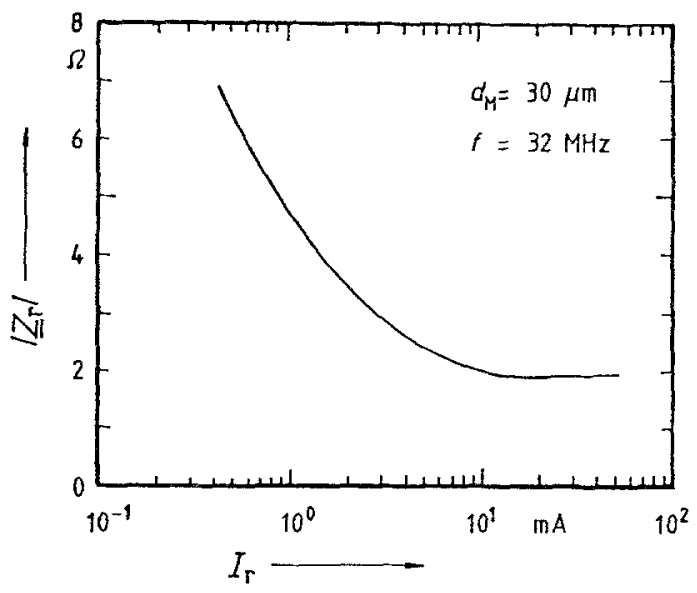

Fig. 7: Absolute value of the small signal diode impedance $\underline{Z}_{r}$ at breakdown as a function of the bias current $I_{B}$. 
standard CV-profiling and precise electrochemical profiling ${ }^{19}$ using the Polaron PN 4200.
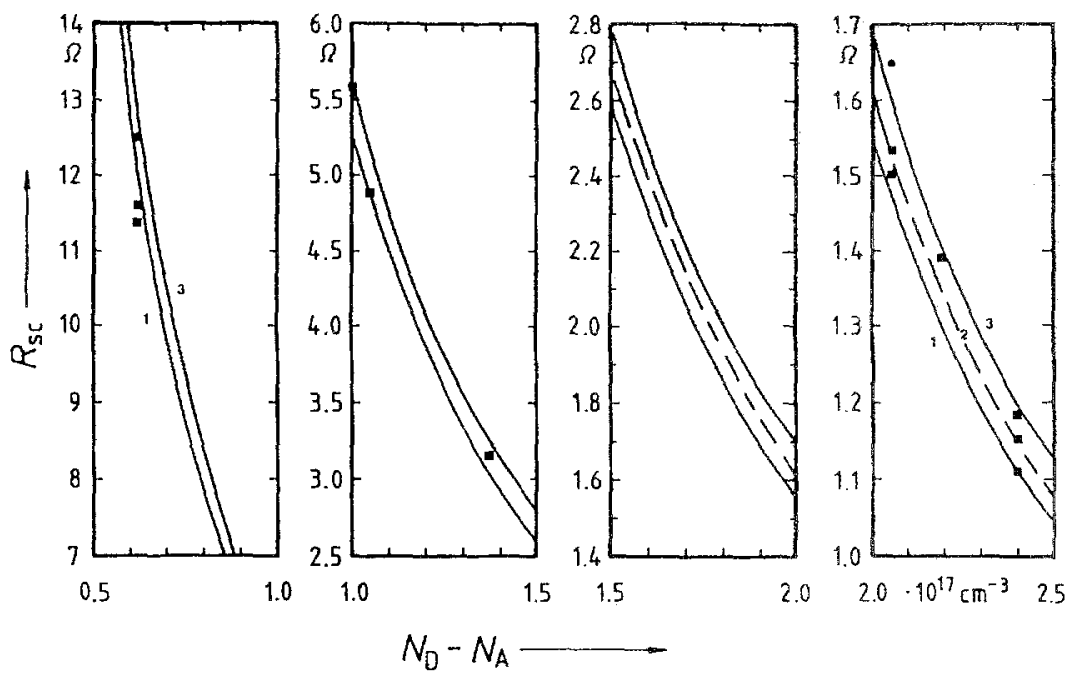

Fig. 8: Space charge resistance $R_{\text {ec }}$ vs. doping concentration $\left(N_{D}-N_{A}\right)$ of the active n-doped layer at $T=300 \mathrm{~K}$, for $A_{D}=1 \times 10^{-5} \mathrm{~cm}^{-2}, I_{B}=$ $20 \mathrm{~mA}$ and the three $v_{d n}(E)$-profiles $1,2,3$ of Fig. 1 .

a : measured values, plotted as $R_{s c} \pi d_{M}^{2} / 4 \times 10^{-5} \mathrm{~cm}^{-2}$ (d $d_{M}$ : diode diameter).

From the curve 2 for $T_{0}=300 \mathrm{~K}$ in Fig. 8 the drift velocity at $T=500 \mathrm{~K}$ has been extrapolated and used together with the extrapolated ionization rates for the design of W-band single-drift flat-profile IMPATT diodes. As state-of-the-art in $\mathrm{GaAs}$ these diodes delivered an output power up to $320 \mathrm{~mW}$ at an efficiency of $6.0 \%$ for an oscillation frequency about $95 \mathrm{GHz}^{22}$.

\section{Conclusion}

The excellent agreement of measured and calculated breakdown voltages for the abrupt pn-junction of GaAs single-drift tlat-profile IMPATT diodes justify the extrapolation of well known ionization rates to electric fields up to about $850 \mathrm{kV} \mathrm{cm}^{-1}$. A straightforward method to calculate space-charge resistances implies field dependent drift velocities of electrons and holes. For the first time, this method is capable to give a clue for the velocity vs. field profile of electrons up to $800 \mathrm{kVcm}^{-1}$. The good agreement between these experimental results and theoretical curves confirm those results obtained from Monte-Carlo simulations. Both results, ionization rates and drift velocities, are a useful starting-point in designing IMPATT diodes for frequencies above $100 \mathrm{GHz}$. 


\section{Acknowledgments}

Most of the measurements and calculations were performed at the Lehrstuhl für Allgemeine Elektrotechnik und Angewandte Elektronik, Technical University Munich. The work was partially supported by the Frauenhofer Gesellschaft. Special thanks go to Xiangkun Zhang for making the IMPATT diodes and results of his thesis available to me. The author thanks Helmut Grothe for supplying the high-quality MBE material. Discussions with Prof. Harth are gratefully appreciated.

\section{References}

1. Eisele, H.: "Selective etching technology for $94 \mathrm{GHz}$ GaAs IMPATT diodes on diamond heat sinks", Solid-State Electronics, 32, 1989, pp. 253-257.

2. Ruch, J. G., Kino, G. S., “Transport Properties of GaAs”, Phys. Rev., 174, 1968, pp. 921-931.

3. Houston, P. A., Evans, A. G. R., "Electron drift velocity in n-GaAs at high electric fields", Solid-State Electron., 20, 1977, pp. 197-204.

4. Smith, P. A., Inoue, M., Frey, J., "Electron velocity in Si and GaAs at very high electric fields", Appl. Phys. Lett., 37, 1980, pp. 797-798.

5. Windhorn, T. H., Roth, T. J., Zinkiewicz, L. M., Gaddy, O. L., Stillman, G. E., "High field temperature dependent drift velocities in GaAs", ibid., 40, 1982, pp. 513-515.

6. Okamoto, H., Ikeda, M., "Measurement of the Electron Drift Velocity in Avalanching GaAs Diodes", IEEE Trans. Electron Devices, ED-23, 1976, pp. 372-374.

7. Sze, S. M., Physics of Semiconductor Devices, 2nd Ed., J. Wiley \& Sons, New York, 1981, pp. 1-868.

8. Glover, G. H., Tantraporn, W., "Doping profle measurements from avalanche space-charge resistance: a new technique", J. Appl. Physics, 46, 1975, pp. 867-874.

9. Shichijo, H., Hess, K., "Band-structure-dependent transport and impact ionization in GaAs", Phys. Rev. B, 23, 1981, pp. 4197-4207.

10. Lippens, D., private communication.

11. Fischetti M. V., Laux S. E., "Monte Carlo analysis of electron transport in small semiconductor devices including band-structure and space-charge effects", Phys. Rev. B, 38, 1988, pp. 9721-9745.

12. Eisele H., "GaAs W-band IMPATT diodes - the first step to higher frequencies", to be published in Microwave Journal, 1990. 
13. Bulman, G. E., Robbins, V. M., Stillman, G. E., "The Determination of Impact Ionization Coefficients in (100) Gallium Arsenide Using Avalanche Noise and Photocurrent Multiplication Measurements", IEEE Trans. Electron Devices, ED-32, 1985, pp. 2454-2466.

14. Okuto, Y., Crowell, C. R., "Threshold energy effects on avalanche breakdown voltage in semiconductor junctions," Solid-State Electron., 18, 1975, pp. 161-168.

15. Holway, L. H., Steele S. R., Adlerstein, M. G., "Measurement of electron and hole properties in p-type GaAs", Proc. of the Seventh Biennial Cornell Electrical Engineering Conference, 1979, pp. 199-208.

16. Brennan, K., Hess, K., "Theory of high field transport of holes in GaAs and InP", Phys. Rev. B, 29, 1984, pp. 5581-5590.

17. Haitz, R. H., Stover, H. L., Tolar, N. J., "A method for heat low resistance measurements in avalanche diodes", IEEE Trans. on Electron. Devices, ED-16, 1969, pp. 438-444.

18. Eisele, H., "Theorie, Technologie und Untersuchung von Lawinenlaufzeitdioden aus MBE-GaAs für W-Band Frequenzen", Ph.D. Thesis, Technical University of Munich, 1989.

19. Eisele, H., "Reproducible and accurate electrochemical doping profile measurements in $\mathrm{GaAs}^{\prime}$, to be published in Archiv der elektr. Übertragung, 1990.

20. Zhang, X., "Herstellung und Untersuchung von GaAs Lawinenlaufzeitdioden für $60 \mathrm{GHz}$ ", Ph.D. Thesis, Technical University of Munich, 1984.

21. Zhang, X., private communication.

22. Eisele H., Grothe H., "GaAs W-band IMPATT diodes made from MBE material", Proceedings of the MIOP ' 89 , Sindelfingen, Feb. 28 - March 2 1989, Session 3A.6. 